\title{
TRM Array Antenna Failure Compensation Using Extended PSO
}

\author{
Singh Gurpreet, Singh Maninder \\ Researcher, ECE, Rayat Institute of Engineering \& Information Technology, Railmajra, S.U.S Nagar, Punjab \\ Assistant Prof., ECE, Rayat Institute of Engineering \& Information Technology, Railmajra, S.U.S Nagar, \\ Punjab
}

\begin{abstract}
TRM array antenna is used in wireless communication system. There are various problem which may occur while the transmission of the data. The biggest problem is of routing. This paper focuses on the optimized routing using PSO which would be compared with the routing of GA. This paper also focuses on the comparison of PSO and GA when compensated to the antennas.
\end{abstract}

Keywords: TRM ARRAY ANTENA, GA, PSO, Wireless Communication.

\section{Introduction}

For wireless communication system, the antenna array is one of the most important components to improve the system capacity and spectral efficiency. The active antenna array is widely used in many applications like satellite communication, sonar, mobile communication etc. for signal acquisition purpose [1].Generally the antenna array consists of large number of radiating elements or sub- arrays. Generally the antenna array consists of large number of radiating elements or sub- arrays. Due to large number of elements presented in an array, there is always a possibility of failure of one or more elements in the antenna array system. The failures of elements in the array destroy the symmetry and may cause sharp variation in intensity across the array, distort the pattern in the form of increased side lobe level. The degradation of the capacity of transmitter/receiver module (TRM) distorts the beam pattern; that is, the side lobe level (SLL) increases [2]. Array processing involves manipulation of signals induced on various antenna elements. Its capabilities of steering nulls to reduce co channel interferences and pointing independent beams toward various mobiles, as well as its ability to provide estimates of directions of radiating sources, make it attractive to a mobile communications system designer. Array processing is expected to play an important role in fulfilling the increased demands of various mobile communications services [3]. Antenna Array is formed by assembly of radiating elements in an electrical or geometrical configuration. In most cases the elements are identical. Total field of the antenna array is found by vector addition of the fields radiated by each individual element. There are five controls in an antenna array that can be used to shape the pattern properly, they are, the geometrical configuration (linear, circular, rectangular, spherical etc.) of the overall array, relative displacement between elements, excitation amplitude of the individual elements, excitation phase of the individual elements and relative pattern of the individual elements. In many communication applications it is required to design a highly directional antenna. Array antennas have a high gain and directivity compared to an individual radiating element. A linear array has all its elements placed along a straight line, with a uniform relative spacing between elements. The goal in antenna array geometry synthesis is to determine the physical layout of the array that produces the radiation pattern that is closest to the desired pattern [4-6]. Today a lot of research on antenna array is being carried out using various optimization techniques to improve null performance due to their robustness and easy adaptively. One among them is Genetic Algorithm, a method for null control and their effects on the radiation pattern [7] [8]. A genetic algorithm (GA) is a method for solving both constrained and unconstrained optimization problems based on a natural selection process that mimics biological evolution. The algorithm repeatedly modifies a population of individual solutions. At each step, the genetic algorithm randomly selects individuals from the current population and uses them as parents to produce the children for the next generation. Over successive generations, the population "evolves" toward an optimal solution. Multiple antenna structures can be divided into three groups: use of antenna array only at receiver, known as single-input multiple-outputs (SIMO) systems; use of antenna array only at transmitter, known as multiple-inputs single-output (MISO) systems; and use of antenna arrays at both transmitter and receiver, known as multiple-inputs multiple-outputs (MIMO) systems.

GA is one of the most advance techniques which is getting used world wide web for each and every purpose. In the same manner GA has been used for the optimization of the TRM antenna failure also. In this work, comparison of GA with PSO is done (Partial swarm optimization) which is one the finest old techniques in the same contrast taken place. 


\section{Array Antenna TRM Failure Compensation}

During the operation of an array antenna system, TRM failure can occur at any time. When a TRM failure occurs, the TRM is supposed to be turned off. When recalculation is done with the remaining TRMs, a resynthesized beam pattern is constructed within a beam pattern mask. Then, the newly calculated amplitude and phase distributions are reset. In TRM Antenna Transmission using MIMO system reduces bit error rate has been challenge from decade. In addition to this, if any receiving antennas get damaged, it is difficulty from a routing table to generate a routing set which is feasible without damaged antenna. Our basic problem is to generate a routing table by using GA.

To solve the problem a virtual node concept which would be activated if any of the receiving antenna is highly heated up. This virtual node will receive signal instead of that heated antenna until and unless the heated antenna get cooled. We start with static routing. If congestion occurs, we call for GA .If the node selected by the GA consumes less energy than the conventional path, then we add the path to the routing environment, else we choose for next path as shown in Fig.1

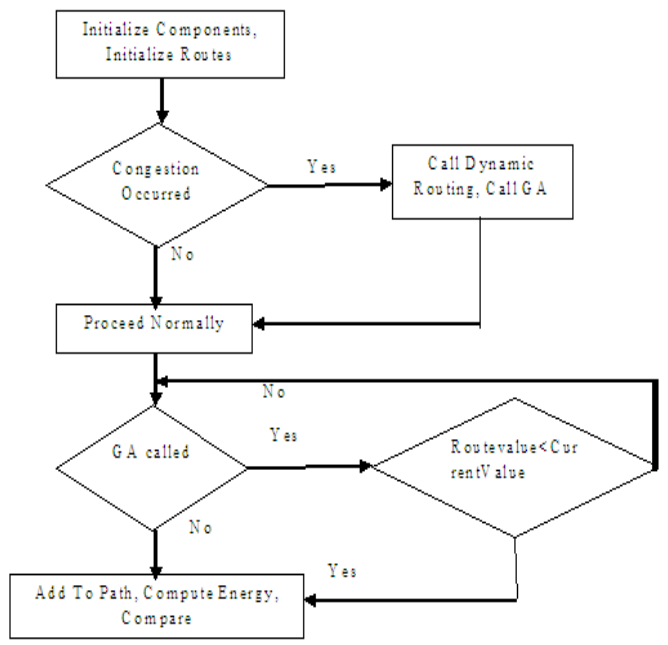

Fig1. Flowchart explaining the method used in the proposed algorithm.

\section{Particle Swarm Optimization ( PSO)}

PSO algorithm is a new intelligent optimization algorithm intimating the bird swarm behaviors, which was proposed by psychologist Kennedy and Dr. Eberhart in 1995 [16]. In PSO algorithm, each individual is called "particle", which represents a potential solution. The algorithm achieves the best solution by the variability of some particles in the tracing space. The particles search in the solution space following the best particle by changing their positions and the fitness frequently, the flying direction and velocity are determined by the objective function.

\section{A. PSO ALGORITHM}

Assuming $\mathrm{Xi}=(\mathrm{xi} 1, \mathrm{xi} 2, \ldots \mathrm{xiD})$ the position of $i$-th particle in $\mathrm{D}$ - dimension, $\mathrm{V}_{\mathrm{i}}=\left(\mathrm{vi}_{1}, \mathrm{vi}_{2}, \ldots, \mathrm{vi}_{\mathrm{D}}\right)$ is its velocity which represents its direction of searching. In iteration process, each particle keeps the best position pbest found by itself, besides, it also knows the best position gbest searched by the group particles, and changes its velocity according two best positions. The standard formula of PSO is as follows:

$$
\begin{gathered}
v_{i d}{ }^{k+1}=w v_{i d}{ }^{k}+c_{1} r_{1}\left(p_{i d}-x_{i d}{ }^{k}\right)+c_{2} r_{2}\left(p_{g d}-x_{i d}{ }^{k}\right) \\
x_{i d}{ }^{k+1}=x_{i d}{ }^{k}+v_{i d}{ }^{k+1}
\end{gathered}
$$

In which $\mathrm{i}=1,2, \ldots \mathrm{N}, \mathrm{N}$-the population of the group particles $; \mathrm{d}=1,2, \ldots \mathrm{D}$; $\mathrm{k}$-the maximum number of iteration; ,the random values between [0,1], which are used to keep the diversity of the group particles; c1,c2; the learning coefficients, also are called acceleration coefficients; $\mathrm{v}_{1 \mathrm{~d}}{ }^{\mathrm{k}}$-the number $\mathrm{d}$ component of the position of particle $i$ in $k$-th iterating; pid, the number $\mathrm{d}$ component of the best position particle $\mathrm{i}$ has ever found; pgd, the number $\mathrm{d}$ component of the best position the group particles have ever found.

The procedure of standard PSO is as following:

1) Initialize the original position and velocity of particle swarm; 
2) Calculate the fitness value of each particle;

3) For each particle, compare the fitness value with the fitness value of pbest, if current value is better, then renew the position with current position, and update the fitness value simultaneously;

4) Determine the best particle of group with the best fitness value, if the fitness value is better than the fitness value of gbest, then update the gbest and its fitness value with the position;

5) Check the finalizing criterion, if it has been satisfied, quit the iteration; otherwise, return to step 2).

\section{FITNESS FUNCITON OF PSO:}

\section{P_FIT $=\operatorname{Min}\left(\operatorname{sqrt}\left(A(x)^{\wedge} 2-B(x)^{\wedge} 2\right)\right)$;}

Where B is the energy at next node and A is the energy of first block.

\section{B. SNR (SIGNAL TO NOISE RATIO)}

Short for signal-to-noise ratio, the ratio of the amplitude of a desired analog or digital data signal to the amplitude of noise in a transmission channel at a specific point in time. SNR is typically expressed logarithmically in decibels $(\mathrm{dB})$.

SNR measures the quality of a transmission channel or an audio signal over a network channel. The greater the ratio, the easier it is to identify and subsequently isolate and eliminate the source of noise. A SNR of zero indicates that the desired signal is virtually indistinguishable from the unwanted noise.

\section{C.BER (BIT ERROR RATE)}

Short for bit error rate, in a digital transmission, BER is the percentage of bits with errors divided by the total number of bits that have been transmitted, received or processed over a given time period. The rate is typically expressed as 10 to the negative power. For example, four erroneous bits out of 100,000 bits transmitted wold be expressed as $4 \times 10-5$, or the expression $3 \times 10-6$ would indicate that three bits were in error out of $1,000,000$ transmitted. BER is the digital equivalent to signal-to-noise ratio in an analog system.

\section{Results And Discussion}

A work is performed with help of dynamic routing using GA (Genetic algorithm).The main concept is to save the energy. Antenna system provide a better performance in wireless network system .First the static routing in case the path is define, data transfer through a selected path in which one node is off mode data is not send .So a dynamic routing in which a dynamic path is selected to one node is off mode the choose nearst node and data transfer a destination, some limition in dynamic routing .So dynamic routing use the GA.

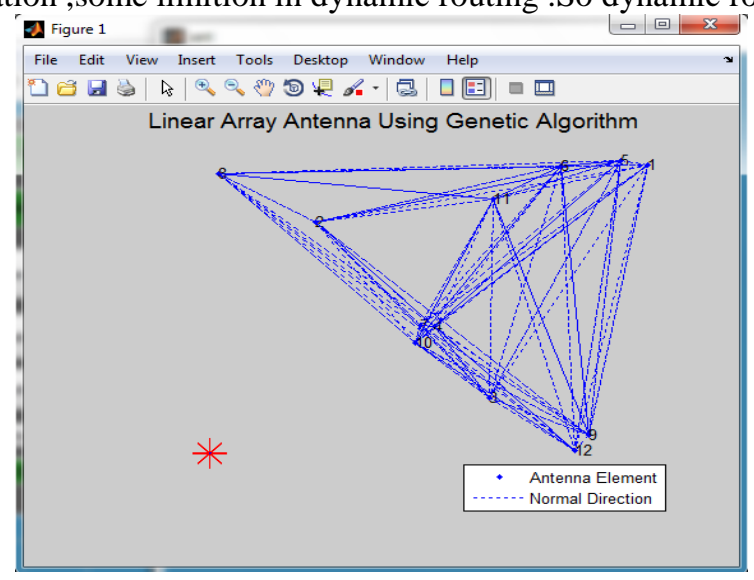

Fig.2 12 Antenna Network

Above figure shows the 12 antenna network,the blue dots are antena element and the blue doted lines are the normal direction of antenna element.These are connected to each other. 


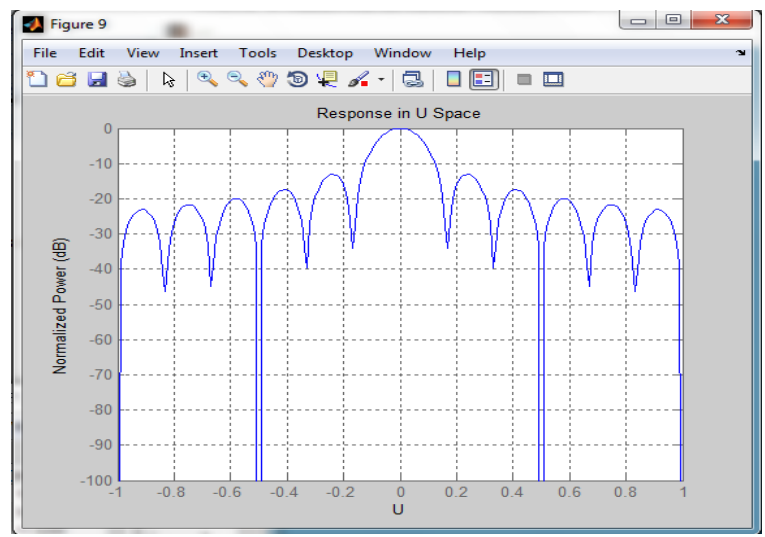

Fig. 3 The Signal of 12 Antenna Systems

The above figure shows 12 antenna signals, the above middle signal is the main signal and side Signals is wastage of signal SLL (side lobe level).

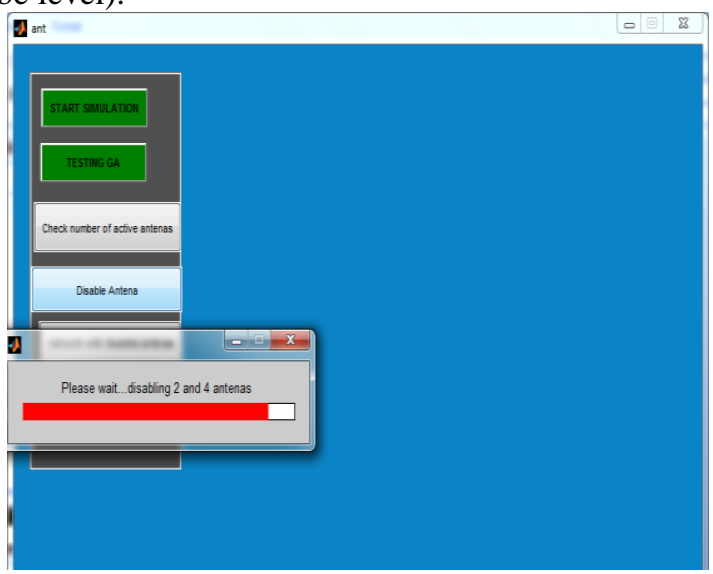

Fig. 4 Disable2 and 4 Antennas

The above figure 2 and 4 antennas are disabled, first generate 10 antenna \& second is 8 antenna system reduce from12, in which the SLL is decreased.

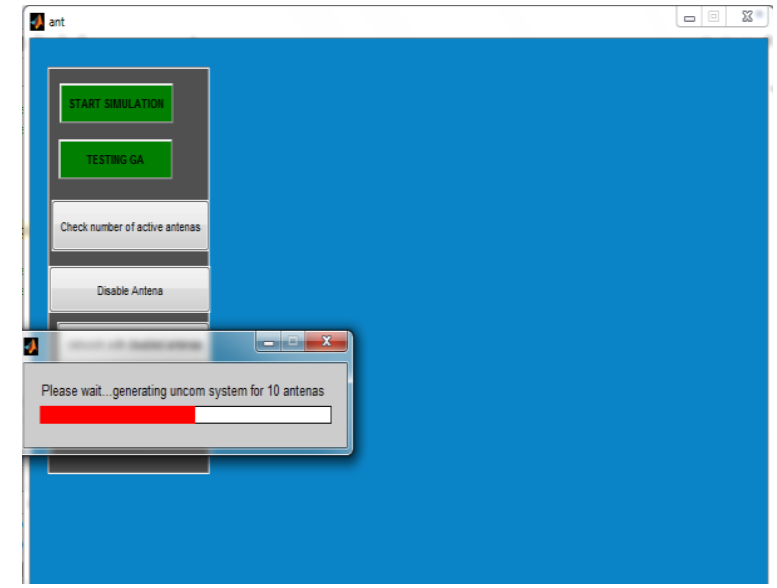

Fig. 5 Generating the Uncomp System for 10 Antenna

The above figure represents the 10 antenna system. The SLL compare to the 12 antenna systems is reduced. 


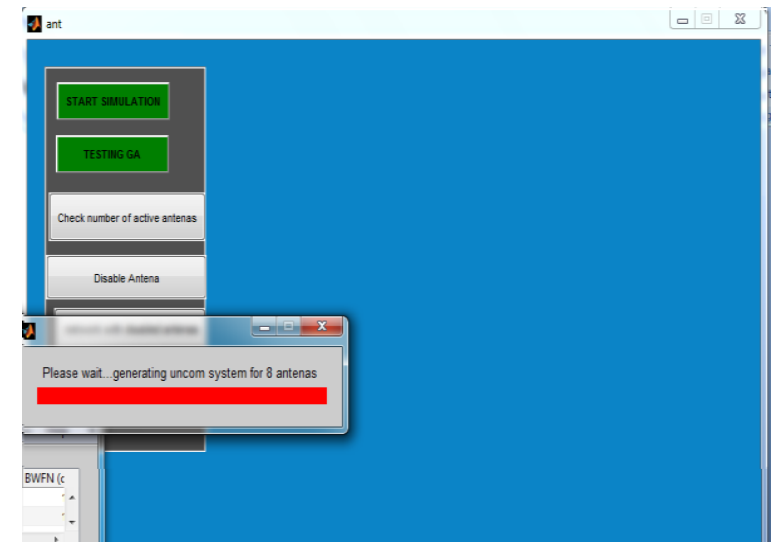

Fig.6 Generating Uncomp System For 8 Antenna

These represents 8 antenna system reduce from 12 antenna system.

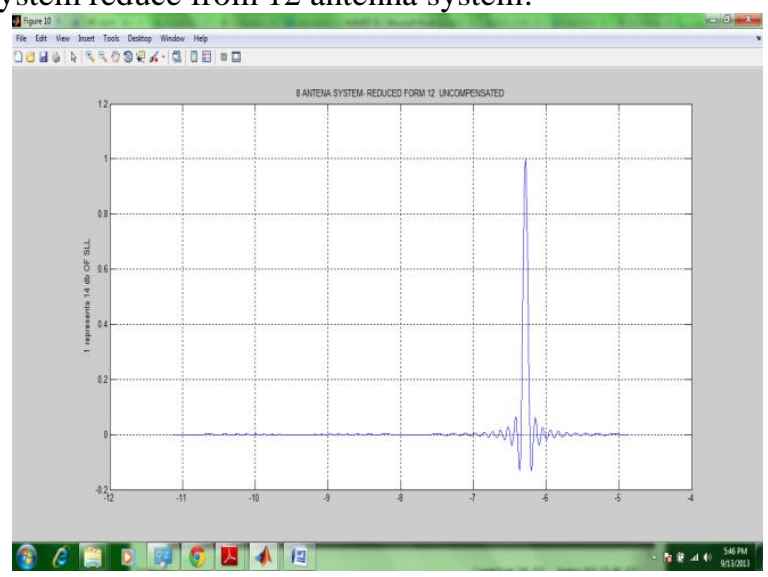

Fig.7 uncompensated structure using the PSO algorithm

The figure shown above describes the side lobe level of the signal whose units have been described on their axis .This is the uncompensated structure using the PSO algorithm. The y axis is the side lobe level and the $\mathrm{x}$ axis represents the deviation of angle. At the y axis, .1 represents $14 \mathrm{db}$ of sll.

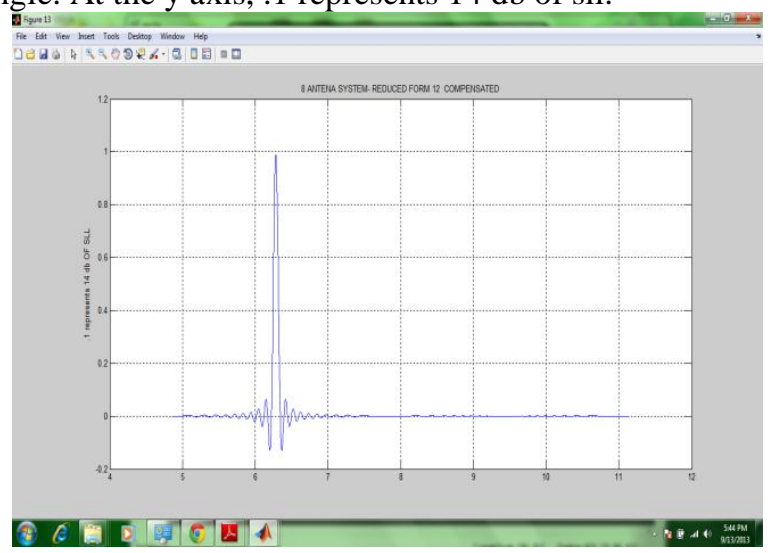

Fig.8 Compensated structure using the PSO algorithm

The figure shown above describes the side lobe level of the signal whose units have been described on their axis .This is the compensated structure using the PSO algorithm. The $\mathrm{y}$ axis is the side lobe level and the $\mathrm{x}$ axis represents the deviation of angle. At the y axis, .1 represents $14 \mathrm{db}$ of sll. 


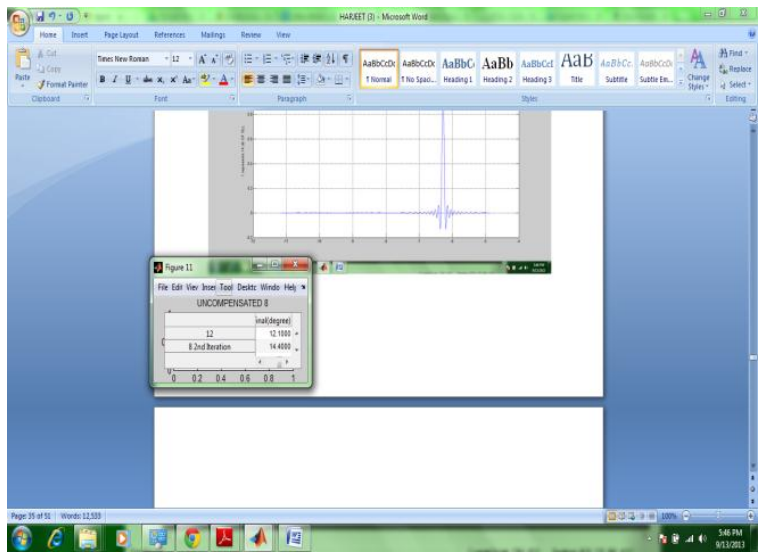

Fig.9 SLL decreases as compare to 12 antennas

Above figure shows 10 uncomp antenna systems reduce from 12 antenna systems. The middle signals is main signal and side signals is the wastage of signal (SLL). These SLL is decrease as compare of 12 antenna.

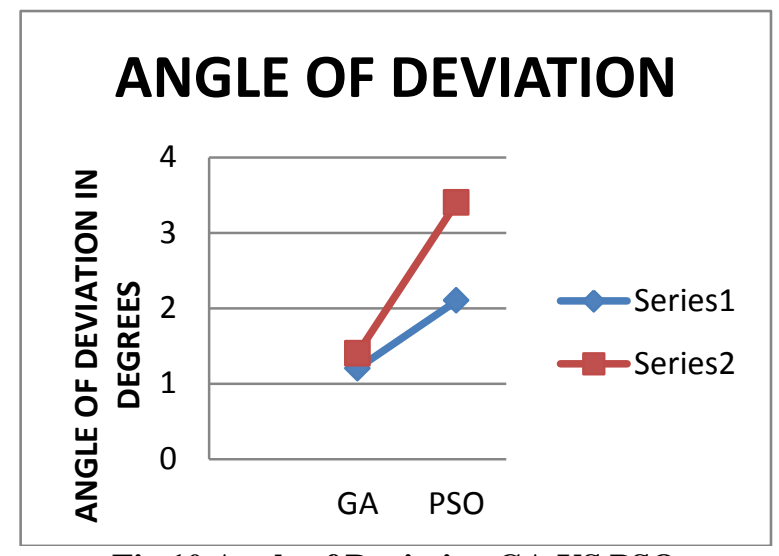

Fig.10 Angle of Deviation GA VS PSO

Angle of deviation is a term which is mentioned to compensate the value of side lobe level. More the value of the side lobe level, less would be angle of deviation and vice versa. More deviation means less side lobe level values. Red graph represents the GA and the blue graph represents the PSO

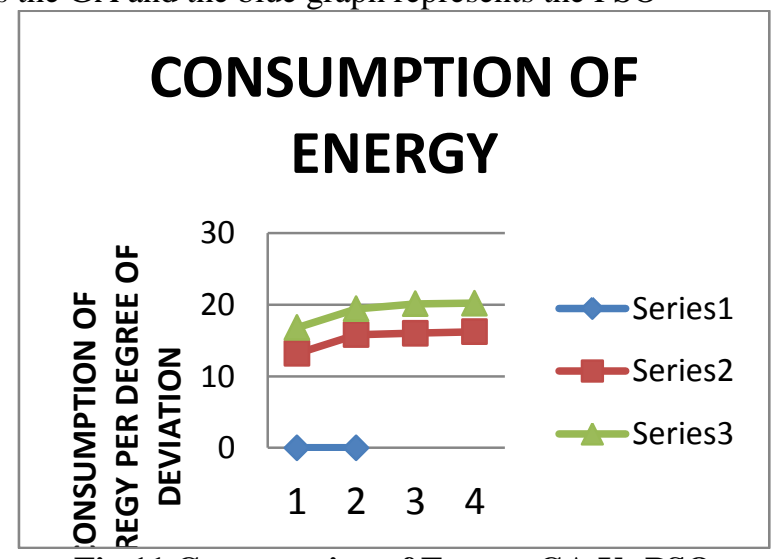

Fig.11 Consumption of Energy GA Vs PSO

The above graph explains the consumption of energy into the routing protocol environment. It calculates the total energy consumed into getting to the destination node and it has been computed by summing up the energy consumed by each node. The energy consumption shows that how much energy got consumed during the process. According to the experimental results, GA consumes less energy in comparison to the PSO . The red line stands for GA where as the green line stands for PSO. 


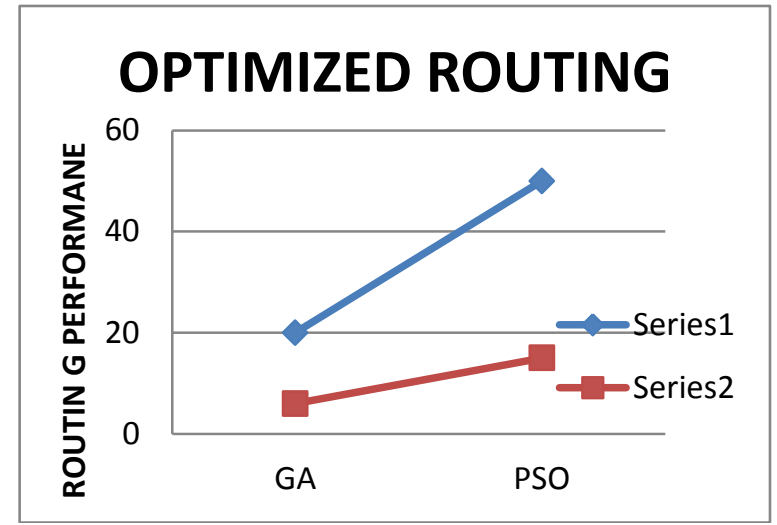

Fig. 12 Optimized Routing GAVs PSO

The above graph shows the optimized performance of the routing for which the research work has been done. According to this graph the routing performance of the GA has been found much better than that of the PSO. GA optimizes the path around $60 \%$ where as PSO sticks to 10 to 15 percent only. The reason behind is this that GA uses fitness and objective function.

\section{Conclusion}

The technology for design and development of a large phased array is presently available However, it has not reached the maturity and low cost levels needed to make it competitive with a reflector antenna system at the present time. It is to believe that in the next 10- 15 years the breakthroughs in mass production techniques at the components, modules and integrated circuit board level will be achieved that will make it an attractive option. There are many advantages to a phased array system as compared to the current $70 \mathrm{~m}$ DSN antenna network. These include high beam agility, multi-beam multi target applications, high reliability and easy maintainability. The disadvantages include much higher cost at the present, complexity of multi-frequency operations, inflexibility in adding new frequencies, and lower gain at lower elevations for a flat horizontal array. The technology risks and the cost drivers include, primarily, the T/R modules and the beamforming network architecture and implementation. It is propose that, as a proof of concept demonstration, a small scalable flat panel array be built and tested, in order to prove the maturity of the concept and to work out the potential problems at the T/R module and the beam-forming levels, for achieving a GA-level performance. This could be a Im-square array at WKa bands. This panel could become an element of a much bigger array composed of such modular elements. The architecture of the connectivity and integration of this panel into a larger system would also be part of the proposed work.GA performance is better.

\begin{tabular}{|l|l|l|}
\hline $\begin{array}{l}\text { RADIATION } \\
\text { VALUE FOR } \\
\text { ANTENNA }\end{array}$ & GA & PSO \\
\hline $\mathrm{N}=12$ & $-13.2 \mathrm{db}$ & $-13.4 \mathrm{db}$ \\
\hline $\mathrm{N}=12$ distorted & $-10.8 \mathrm{db}$ & -11.7 \\
\hline $\mathrm{N}=12$ compensated & $-11.3 \mathrm{db}$ & $-12.6 \mathrm{db}$ \\
\hline
\end{tabular}

The above table represents the radiation pattern structure of 12 array antenna with the distorted and compensated. The table clearly shows that the PSO is better when we provide compensation to the antenna through PSO.

\section{Future Scope}

There are several methods for reflect array elements to achieve the desired planar phase front. The first uses identical micro strip patches with different length phase delay lines attached so that they can compensate for the unequal phase delays due to the differing path lengths from the illuminating feed. Low-loss electronic phase shiftless employing micro-electro-mechanical switches (MEMS) can be inserted into these phase-delay lines to achieve electronic beam scanning. Since the antenna contains a very large number of elements, 2- or 3bit phase shifters with low insertion loss are sufficient to achieve good overall beam scan resolution. The parallel-fed reflection-type phase shifters generally yield lower insertion loss than the conventional series-fed transmission-type phase shifters. The second method, which only works with circular polarization, employs 
identical circularly polarized elements with different angular rotations to compensate for the feed path length differences. Micro-machined motor can be placed underneath each patch to mechanically rotate the element and effect fast beam scanning. With this approach, there is nearly no insertion loss associated with the elements and beam- scanning speeds of the order of milliseconds can be readily achieved. Other than this there are several future generation algorithms which can be combined with GA to optimize the results like, neural networks or fuzzy logics. The future researchers can try their hands on this scenario.

\section{References}

[1]. Narwant S. Grewal, Munish rattan and Manjeet S. Patterh, "A linear antenna array failure correction Using firefly algorithm", progress in electromagnetics research $\mathrm{m}$, vol. 27, 241 \{254, 2012\}.

[2]. Jung-Hoon Han, Sang-Ho Lim and Noh- Hoon Myung, "Array antenna trm failure compensation using Adaptively weighted beam pattern mask Based on genetic algorithm," ieee antennas and wireless propagation letters, vol. 11, 2012.

[3]. Lal c. Godara, "Application of antenna arrays to mobile Communications, part ii: beam-forming And direction-of-arrival considerations," proceedings of the ieee, vol. 85, no. 8, august 1997.

[4]. C. A. Ballanis, "Antenna theory analysis and design," 2nd edition, john willey and son's inc • new york, 1997.

[5] Elliott, R. S., "Antenna Theory and Design", revised edition, john wiley, new jersey, 2003.

[6]. R. L. Haupt, and d. H. Werner, "Genetic algorithms in electromagnetics", IEEE press wiley-interscience, 2007.

[7]. $\quad$ R. L. Haupt, "Optimized element spacing for low sidelobe concentric Ring arrays," IEEE trans. Antennas propag., vol. 56(1), pp. 266-268, Jan. 2008.

[8]. H. Steyskal, r. A. Shore, and r. L. Haupt, "methods for null control And their effects on the radiation pattern," IEEE trans. Antennaspropagat., vol. 34, no. 3, pp. 404-409, mar. 1986.

[9]. Mohammad Ali Khalighi, Kosai Raoof and Geneviève Jourdain, "capacity of wireless communication systems Employing antenna arrays, a tutorial study," wireless personal communications 23: 321-352, 2002. ( 2002 kluwer academic publishers. Printed in the netherlands.

[10]. Michael A. Jensen, "A Review of Antennas and Propagation for MIMO Wireless Communications," IEEE transactions on antennas and propagation, vol. 52, no. 11, november 2004.

[11]. Benjamin Bräutigam, Marco Schwerdt, and Markus Bachmann, "An Efficient Method for Performance Monitoring of Active Phased Array Antennas," IEEE transactions on geoscience and remote sensing, vol. 47, no. 4, april 2009.

[12]. Bipul Goswami and Durbadal MandaI, "Introducing Deeper Nulls and Reduction of Side Lobe Levels in a Symmetric Linear Antenna Array Using Genetic Algorithm," 1 st int'I Conf. on Recent Advances in Information Technology I RAIT-2012 I.

[13]. Arun Ranjitkar and Young-Bae Ko, "A Collaborative Routing for Wireless Multi-hop Networks with Directional Antennas," 978 $1-4244-8086-9 / 10 / \$ 26.00$ @2010 IEEE.

[14]. Alka Verma, "Analysis And Design Of E Shaped Patch Antenna In X Band," International Journal Of Advanced Engineering Technology E-Issn 0976-3945.

[15]. Tomohiko Mitani, Shunji Tanaka, Yoshio Ebihara, "An Efficient Algorithm forTransmitting Power Maximization of Phased Arrays Including Amplitude Degradation," Proceedings of the "2013 International Symposium on Electromagnetic Theory.

[16]. Kennedy, J. and Eberhart, R.C. (1995) P article swarm optimization. IEEE International Conference on Neural Network, $1942-$ 1948. 